\title{
Clinical Outcomes of Colorectal Cancer in Kenya
}

\author{
Authors: Saidi $\mathbf{H}^{\mathbf{1}, 2}$ MBChB, MMed, FACS, Abdihakin $\mathbf{M}^{\mathbf{2}}$ MBChB, Njihia $\mathbf{B}^{\mathbf{1}}$ Bsc, Jumba $\mathbf{G}^{\mathbf{1}}$ Phd, Kiarie $\mathbf{G}^{\mathbf{1}}$ MMed, Githaiga $\mathrm{J}^{\mathbf{1}}$ MMed, Abinya \\ NO ${ }^{1,2}$ MMed. Affiliations: 1-University of Nairobi and Kenyatta National Hospital 2-Aga Khan University Hospital, Nairobi Correspondence: \\ Prof. Hassan Saidi, Dept of Human Anatomy, University of Nairobi. P.0. Box 30197, GP0 00100, Nairobi, Kenya. E-mail hsaidi@uonbi.ac.ke
}

\section{Summary}

\section{Background}

The incidence of colorectal cancer in Africa is increasing. True data on clinical outcomes of the disease is hampered by follow up challenges.

\section{Method}

Follow up data of 233 patients treated for colorectal cancer between 2005 and 2010 at various Nairobi hospitals were evaluated. The primary outcome was mortality while secondary outcomes included recurrence rates, time to recurrence and the patient, disease and treatment factors associated with mortality and recurrence. Kaplan Meir charts were charted for survival trends.

Results

Half of the lesions were located in the rectum. There was no relationship between the sub-site location and recurrence and mortality.

\begin{abstract}
The mean follow-up period was 15.9 months. Overall recurrence and mortality rates were $37.5 \%$ and $29.4 \%$ respectively. Most recurrences occurred within one year of surgery. Recurrence was not influenced by age, gender, sub-site, chemotherapy receipt or presence of comorbidity. Factors significantly associated with mortality included the male gender ( $p 0.04)$, presence of co-morbidity ( $p 0.029)$, recurrence ( $p$ 0.001), curative intent ( $p$ 0.01), disease stage ( $p 0.036)$ and receipt of chemotherapy $(p<0.01)$.

\section{Conclusion}

Follow up of colorectal cancer patients is still challenging. The mortality and recurrence rates are high for the short follow up periods. Further studies are needed to explore the determinants of both survival and recurrences, especially with longer follow ups.
\end{abstract}

\section{Introduction}

The incidence of colorectal cancer (CRC) in the developed countries is stable or decreasing (1) while that in Africa is increasing $(2,3)$. African CRC presents in late stages and in relatively younger patients. Outcome studies from America suggest worse outcomes for African Americans in comparison to the whites (1). Worse treatment outcomes are tied to treatment access, screening practice, and presence and nature of co-morbidities (1). In an earlier Kenyan study, mortality in CRC was worse for men and emergency surgery (2). The follow-up challenges are major and the true clinical outcomes remain largely unknown.

We sought to further define CRC outcomes and relate these to patient, tumor and treatment variables

\section{Patients and Methods}

This was a chart review of incident cases treated between 2005 and 2009 and followed up by the authors (HS, GJ, GK, JG, NOA) at the Kenyatta National and the Aga Khan University hospitals in Nairobi. A smaller group accrued from private offices of the authors, having had their treatments at other hospitals in Nairobi. Only pa- tients with sufficient information on CRC pathology, treatment and follow up were included. Patient profile, tumor sub-site, pathology details, recurrence and mortality data were collected.

The primary outcome was CRC mortality. Secondary outcomes included rate of recurrences, time to mortality and recurrence and the associated patient and disease factors.

Means (SD) or frequencies (\%) for distributions were calculated. Outcome groups were compared for continuous and categorical variables using Students t-test and $\mathrm{X}^{2}$ as appropriate with significance level set at $\mathrm{p}<0.05$. Survival trends were charted using the Kaplan Myer method.

\section{Results}

Two hundred and thirty three patients were studied. Fifty eight others were excluded for lack of key data elements. The proportion of black African patients was 91\%. Males comprised $58.8 \%$ of the group while the proportion with co-morbid diseases was $38.3 \%$. The peak age (25.9\%) affected was 41-50 years (all-group mean age 53 years). The proportion of patients 40 years of age or younger was $17.6 \%$ (Fig 1). 
Half $(50.5 \%)$ of the lesions were located in the rectum (rectosigmoid lesions comprised $63.3 \%$ of all cases) (Fig. 2). There was no relationship between tumor subsite location and age, gender, recurrence and mortality. The surgical procedures undertaken and chemotherapy regimens administered are shown in table 1 . The mean follow-up period was 15.9 months (range 1-20 months). Overall recurrence and mortality rates were $37.5 \%$ and 29.4\% respectively. Most recurrences occurred within one year of the surgery (fig. 3). Recurrence was not influenced by age, gender, sub-site, chemo receipt, and comorbidity status.

\section{Pathology}

Most lesions (70\%) were well or moderately differentiated. The disease stage at treatment was I (7\%), II (22.7\%), III (40.1\%) and IV (29.1\%).

For a variable proportion of reports, key data elements were missing. Staging data was missing in $22.9 \%$ of reports, differentiation unstated in 52.9\%, lympho-vascular status not shown in $41.3 \%$ of reports and nodal characterization absent in $37.2 \%$. In $34.3 \%$ of reports, the nodal harvest was inadequate (less than 12 nodes harvested).

\section{Statistical Analysis}

On univariate analysis, the factors that were significantly associated with mortality included the male gender, presence of co-morbidity, recurrence, receipt of chemotherapy, disease stage and curative intent (table 3 ). The following factors did not influence mortality: post-operative morbidity (p 0.08), age (p 0.55), sub-site (p 0.86), race ( $\mathrm{p} 0.47$ ), tumor differentiation ( $\mathrm{p} 0.33$ ), and type of chemotherapy ( $p$ 0.22). The Kaplan Meir curves depicting survival trends are shown in figure $4 \mathrm{a}-\mathrm{d}$.

\section{Discussion}

The results of the current study have provided additional outcome data on colorectal cancer in Kenya. The data on young age and advanced stage at presentation corroborate earlier data from the region $(2,4,6)$. The youngest patient in this group was 18 years with peak age of 41-50 years. In Lagos and Sagamu in Nigeria (6) the youngest was 10 years with a peak of 60-69 (mean 50.7 and 23\% younger than 40 years).

The pattern of young age of presentation contrasts West-

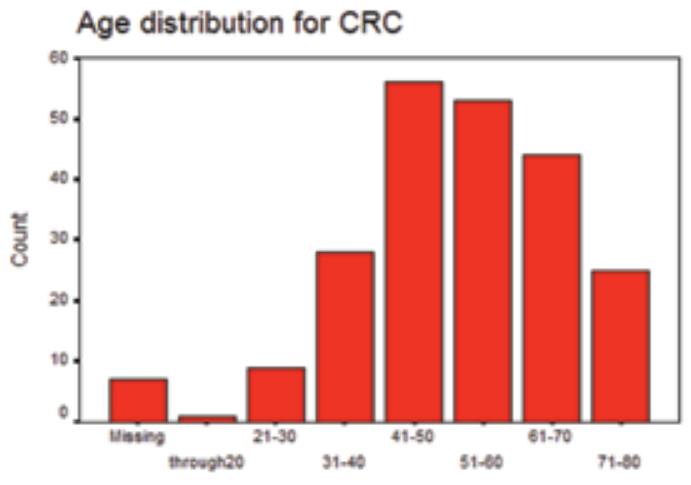

Age groups

Fig.. 1: Age distribution of CRC

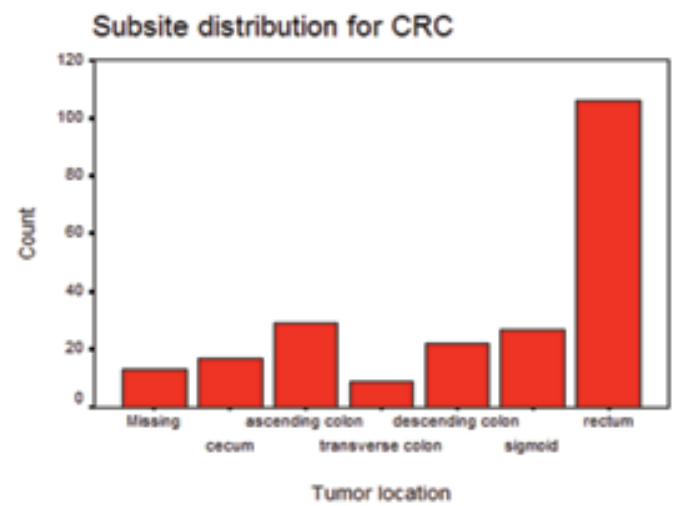

Fig. 2: Sub-site distribution of CRC

\begin{tabular}{lll} 
Surgical Procedure & Frequency & \% \\
\hline Right hemiclectomy & 43 & 22.9 \\
\hline Transverse colectomy & 3 & 1.6 \\
\hline Left hemicolectomy & 33 & 17.6 \\
\hline Sigmoid colectomy & 3 & 1.6 \\
\hline Anterior resections & 22 & 11.7 \\
\hline Abdominoperineal resection & 54 & 28.7 \\
\hline Colostomy/bypass/no surgery & 30 & 15.9 \\
\hline Chemotherapy Regimen & Frequency & $\%$ \\
\hline Folfox & 33 & 29.2 \\
\hline Xelox & 9 & 7.9 \\
\hline Folfiri & 2 & 1.8 \\
\hline Xeloda & 8 & 7.1 \\
\hline Avastin & 1 & 0.9 \\
\hline Irinotecan & 1 & 0.9 \\
\hline 5 FU \& Leucovorin & 59 & 52.2 \\
\hline
\end{tabular}

Folfox- 5FU-leucovorin/oxaliplatin combination, Xelox - oral capecitabine/oxaliplatin combination, Folfiri- 5FU-leucovorin/irinotecan combination, Xeloda - oral capecitabine, avastin - bevazixumab

Table1: list of surgical procedures performed and chemotherapeutic agents used 
Clinical Outcomes of Colorectal Cancer in Kenya

Saidi H, Abdihakin M, Njihia B, Jumba G, Kiarie G, Githaiga J, Abinya N0

\begin{tabular}{llll} 
Variable & Mortality & Survival & Pvalue \\
\hline Male & 42 & 79 & 0.04 \\
\hline Female & 16 & 60 & \\
\hline Co-morbids & 20 & 27 & 0.029 \\
\hline No co-morbids & 36 & 104 & \\
\hline Recurrence & 23 & 29 & 0.001 \\
\hline No recurrence & 16 & 72 & \\
\hline Curative intent & 35 & 109 & 0.01 \\
\hline Palliative intent & 8 & 7 & \\
\hline Stage I/II & 8 & 40 & 0.036 \\
\hline Stage I//IV & 35 & 71 & \\
\hline Chemotherapy given & 31 & 103 & $<0.001$ \\
\hline Chemotherapy not given & 26 & 22 & \\
\hline
\end{tabular}

Table 2: Factors associated with mortality after CRC

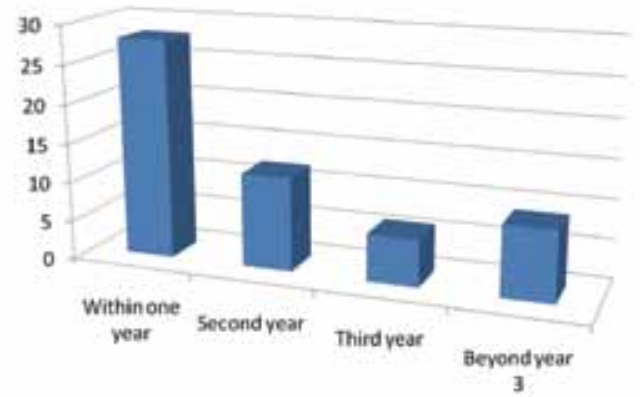

Fig. 3. Time to recurrence in Kenyan CRC

ern data (1). A trend towards younger age at presentation amongst Americans has however been recently reported. Meyer et al, analyzing the surveillance, epidemiology and end results (SEER) database reported a $3.8 \%$ increase in the rate of rectal cancer in the young between 1984 and 2005 but could not demonstrate a similar trend for colon cancer (6). The authors had no explanation for the observation. Future research direction will entail defining this younger population better through genetic and family analyses $(4,6)$.

The advanced stage at presentation also contrasts the presentation amongst Americans, especially the nonblack population. Whereas $29 \%$ of cases in the current study have presented with metastatic disease, in the report by Polite et al. the respective proportions for Americans was $18 \%$ for whites and $24 \%$ for African Americans (1). These differences are reflected in the survival patterns. The three-year overall survival for our local CRC, derived from the survival curves was $40 \%$, in contrast to better rates at even longer follow up, 65\% for whites and $55 \%$ for African Americans at five years . Differing tumor biologies may explain the discrepancies as may factors related to access to diagnosis and treatment (1).

In one of the earlier Kenyan studies, factors that significantly influenced mortality included - gender and emergency surgery. The effect of gender has persisted in the current analysis. Additional factors in the present study included the presence of co-morbidity, recurrence, curative intent, disease stage and use of adjuvant chemotherapy. Our results are comparable to others for the effect of stage and receipt of chemotherapy (7). Further local studies are needed to show the real association with age, race and type of chemotherapy.

The study has additionally shown a number of areas that need improvement in our CRC care pathways. The follow-up period, although 10 months longer than the earlier study (2), attests to the need to develop prospective databases that can more reliably monitor CRC outcomes long term. Pathology reports were discordant with international guidelines in a significant number of reports. A quality monitoring tool, if effected, will ensure concordance with chemotherapy for node positive cases, surgical margins and nodal harvest, median time intervals of receipt of treatment and pathology reporting. The Kenya oncological research database (8) is one such effort designed to improve the care of CRC in Kenya. Using data from this source, care givers can interrogate the follow-up data more thoroughly and analyse survival more comprehensively than the overviews we have documented in this report.

\section{Acknowledgement}

The study results were presented at the East Africa Oncology Experience meeting, Serena Hotel, Mombasa, Kenya April 2010

\section{References}

1. Polite BN, Dignam JJ: A colorectal cancer model of health disparities:Understanding mortality differences in minority populations. J Clin Oncol 24:2179-2187, 2006 


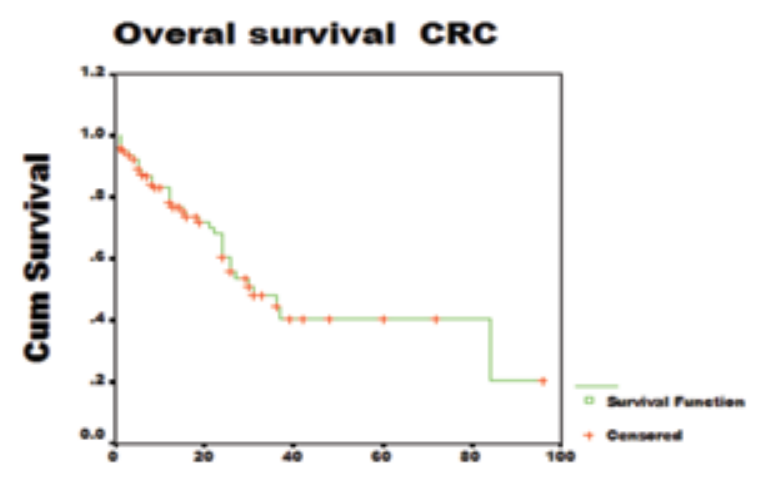

Duration of follow up (months)

Fig. 4a: Overall survival curve in $C R C$

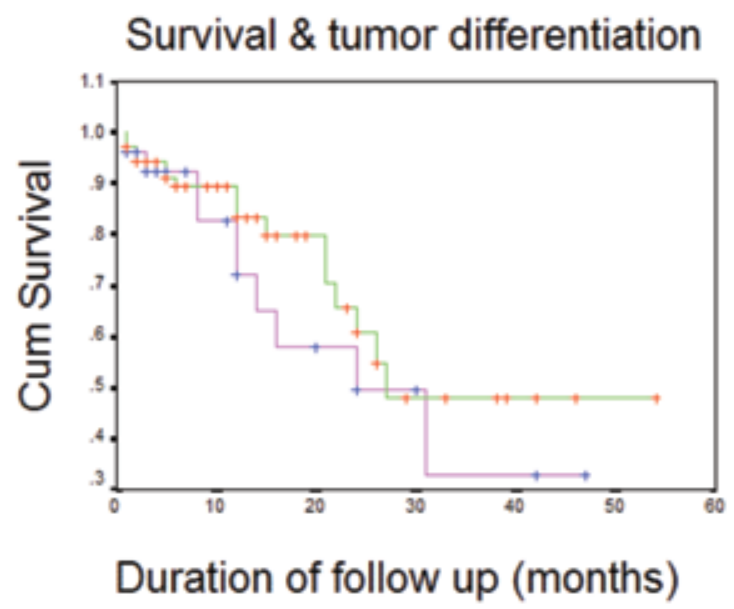

Fig. 4c: survival curve: influence of tumor differentiation

\section{Survival versus stage of disease}

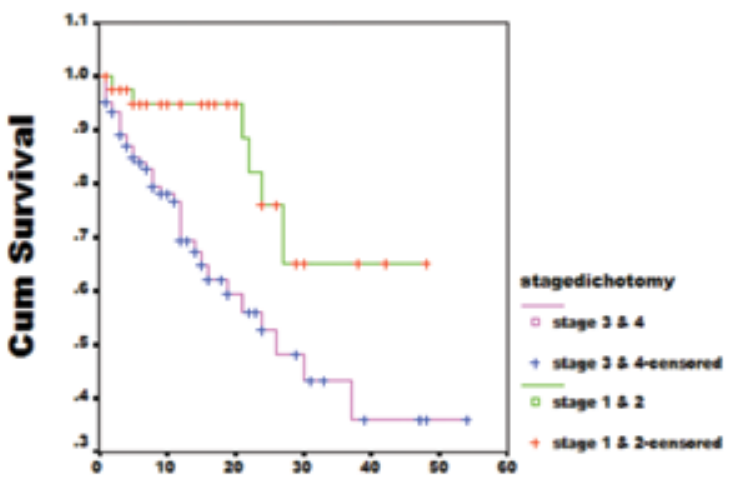

Duration of follow up (months)

Fig. 4b: survival curve: influence of stage

Survival versus chemotherapy reg

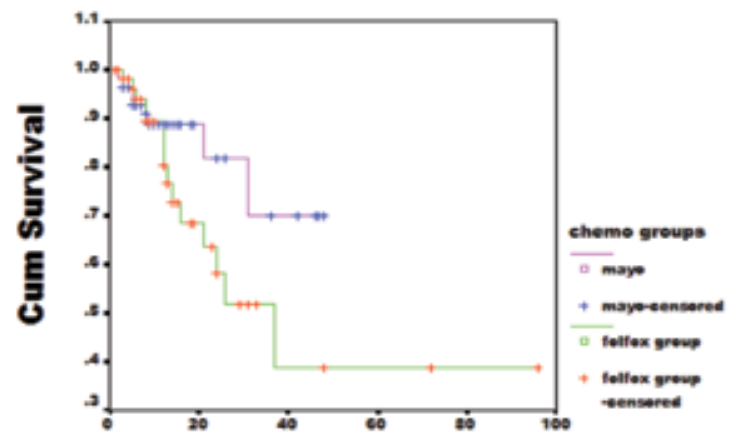

Duration of follow up (months)

Fig. 4d: survival curve; influence of chemotherapy regimen
2. Saidi H, Nyaim EO, Githaiga JW, et al. Colorectal cancer surgery trends in Kenya, 1993-2005. World J of Surg, 2008; 32: 217-223

3. Iliyasu Y, Ladipo JK, Akang EE, et al. A twenty-year review of malignant colorectal neoplasms at University College Hospital, Ibadan, Nigeria. Dis Colon Rectum. 1996 May;39(5):536-40.

4. Saidi H, Nyaim EO, Karuri D, et al.. Young patients with colorectal cancer at a tertiary hospital in Kenya, 1993-2005. Ann Afr. Surg, 2007; 1: 10-15

5. Abdulkareem FB, Abudu EK, Awolola NA, et al. Colorectal carcinoma in Lagos and Sagamu, Southwest Nigeria: a histopathological review. World J Gastroenterol. 2008; 14;(42):6531-5.

6. Meyer JE, Narang T, Schnoll-Sussman FH, et al. Increasing incidence of rectal cancer in patients aged younger than 40 years: an analysis of the surveillance, epidemiology, and end results database. Cancer. 2010 Sept 15 (published online August 23 wileyonlinelibrary.com)

7. Andre T, Boni C, Mounedji-Boudiaf L, et al. Oxaliplatin, fluorouracil, and leucovorin as adjuvant treatment for colon cancer. N Engl J Med 2004;350:2343-2351

8. The Kenya Oncological Research Database, accessed 02 November 2010, <http://www.cancer.co.ke> 